\title{
Distintas competencias en el proceso de conversión del lenguaje natural al algebraico
}

\author{
Carlos Soneira Calvoi, María José Souto Salorioii \& Ana \\ Dorotea Tarrío Tobariii
}

Universidade da Corunha, España

\section{Resumen}

El uso de distintos registros semióticos y el cambio de uno a otro son características de la actividad cognitiva en matemáticas, y las dificultades asociadas a esos cambios fuentes de incomprensión y dificultades de aprendizaje. En el cambio del lenguaje natural al algebraico intervienen al menos dos tipos de competencias: manejar estructuras sintácticas en lenguaje verbal y movilizar mentalmente conceptos matemáticos implícitos en los enunciados. Para estudiar la incidencia de cada una de ellas, las dificultades que presentan y su interrelación, en este trabajo diseñamos un cuestionario, lo aplicamos a un grupo de estudiantes de Ingeniería y analizamos los resultados mediante contrastes de hipótesis, extrayendo conclusiones aplicables al diseño de instrucción. Los resultados muestran diferencias estadísticamente significativas en función tanto del llamado carácter congruente o no del cambio de registro semiótico, como de la complejidad sintáctica del enunciado.

Palabras clave

Lenguaje Verbal; Lenguaje Algebraico; Conversión; Complejidad Sintáctica

\section{Introducción}

En la actividad matemática intervienen distintos registros de representación, como el pictórico, el lenguaje natural, el algebraico, o las 
representaciones físicas. Entendemos estas últimas como concretizaciones materiales de estructuras matemáticas, un ejemplo de las cuales sería el ábaco, que representa la estructura del sistema de numeración posicional regular. Las representaciones semióticas están presentes en varias líneas de investigación en Educación Matemática (Duval, 1993; Golding, 1998b). Aunque asumimos la naturaleza mental del conocimiento matemático y la cognición como actividad realizada mediante representaciones mentales (Kintsch, 1998), la generación de una representación mental se basa en representaciones externas, entre ellas las semióticas (Dreyfus, 1991; Kaput, 1987). En este trabajo, damos a la noción de representación semiótica el mismo significado que Duval (2004), esto es, integrada en un sistema dotado de una estructura que comporta reglas de combinación entre signos, de tal modo que estas tengan sentido y puedan a su vez ser manipuladas.

La noción de sistema o registro semiótico permite a Duval (2006a) una categorización de las representaciones externas. La primera gran división se refiere a las semióticas, que permiten denotar objetos, frente a las nosemióticas, en las que existe una relación de causalidad entre el sistema de representación y el objeto representado. A su vez, las semióticas se pueden clasificar atendiendo a diversas características (Duval, 2006a). Además, se puede definir una correspondencia entre cada representación externa y la competencia cognitiva para construir y manipular esa representación (Golding, 1998a, 1998b).

En el marco teórico que seguimos en este trabajo (Duval, 1993, 2006a, 2006b) se caracteriza la comprensión en matemáticas como la capacidad para manejar simultáneamente diversos registros de representación y pasar de uno al otro con soltura, proceso denominado conversión. Adoptando la mencionada correspondencia interna-externa (Golding, 1998a, 1998b), la concepción de Duval coincide en gran medida con las fases del proceso de aprendizaje en matemáticas propuestas por Dreyfus (1991). Particularizando en la resolución de problemas, Villegas, Castro, y Gutiérrez (2009), mediante un análisis de protocolos de resolución de problemas de optimización, estudian las traducciones entre las representaciones pictórica, simbólica y verbal, que el sujeto realiza durante la resolución, y concluyen que los resolutores más competentes son aquellos más hábiles haciendo traducciones entre varias representaciones. 
Por otro lado, varios autores ponen de manifiesto la relevancia del lenguaje verbal natural en el aprendizaje de las matemáticas. Es el más usado por el docente durante las clases; el estudiante lo usa para comunicar sus ideas y la verbalización del pensamiento propio aporta al estudiante feedback sobre el estado del aprendizaje (Pimm, 1987). Una de las variables a tener en cuenta para estudiar este lenguaje es el nivel de complejidad sintáctica de las estructuras que vertebran mensaje. En el contexto de la resolución de problemas de enunciado verbal, esta complejidad sintáctica es otra variable de la tarea y como tal ha sido estudiada por otros autores (Puig \& Cerdán, 1988; Valentin \& Chap-Sam, 2005), pero en un contexto de resolución de problemas aritméticos y con estudiantes de primaria.

En este trabajo estudiamos desde un punto de vista cognitivo el paso del lenguaje natural al algebraico. Para ello proponemos a un grupo de sujetos la tarea consistente en realizar la conversión de los 20 enunciados verbales de un cuestionario. Analizamos la relación entre la competencia para manejar las estructuras sintácticas del lenguaje natural y la competencia para movilizar mentalmente resultados matemáticos no mencionados explícitamente en el enunciado pero necesarios para obtener la expresión algebraica. Se analizan también las dificultades de la tarea en función de las competencias requeridas. Los resultados serán aplicables al diseño de instrucción.

\section{Registros de representación semiótica en la actividad matemática}

Duval (1993, 1995, 2006a) analiza los procesos de enseñanzaaprendizaje de las matemáticas a través de los procesos cognitivos que tienen lugar durante la actividad matemática. Un conocimiento de los mismos permitirá, a su vez, estudiar las causas de la incomprensión y mejorar los diseños de instrucción. Se establecen tres características de la actividad cognitiva propia de las matemáticas. La primera es la importancia de las representaciones semióticas. Esta reside en la capacidad de sustituir unos signos por otros signos, más que en la sustitución de objetos matemáticos por signos o una mayor brevedad en la notación. Esto condiciona la actividad matemática porque algunos procesos matemáticos requieren un menor esfuerzo cognitivo si se realizan razonando a nivel sintáctico, sin que intervenga la componente semántica. Pensemos por ejemplo en cómputos 
con números grandes, o en el reagrupamiento de las incógnitas y los términos independientes en un sistema de ecuaciones. La segunda característica es la conocida como paradoja de Duval (1993). Los objetos matemáticos no son directamente accesibles mediante la percepción, sólo lo son a través de representaciones semióticas. Pero entonces, “¿Cómo pueden los aprendices evitar confundir los objetos matemáticos con sus representaciones semióticas si sólo pueden relacionarse con ellos mediante representaciones semióticas?" (Duval, 1993, p. 38). Para evitar ambigüedades, tomamos la noción estructuralista de objeto matemático (Shapiro, 1997), que concibe estos objetos como estructuras abstractas o posiciones dentro de otras estructuras (por ejemplo, el número 2 sería una posición en la estructura de los números naturales). La tercera es la gran variedad de representaciones semióticas usadas en matemáticas, siendo algunos procesos más sencillos en un registro que en otro, y otros tan sólo viables en uno.

A nivel operativo distinguimos dos tipos de transformaciones de representaciones semióticas, los tratamientos y las conversiones. Los tratamientos son transformaciones de representaciones dentro del mismo registro, como despejar la incógnita en una ecuación. Las conversiones consisten en cambiar el registro de representación para un mismo objeto representado, como expresar el enunciado de un problema verbal mediante una o varias ecuaciones, o realizar la representación gráfica de una función a partir de su expresión algebraica. Para realizar la conversión hace falta reconocer el objeto matemático representado, distinguiendo los rasgos no relevantes impuestos por el registro fuente, de la información esencial que define el objeto matemático. Sólo esta última debe ser llevada a otro registro. Pero ese reconocimiento implica necesariamente un cierto grado de comprensión global del objeto representado, por lo que "la comprensión (integral) de un contenido conceptual está basada en la coordinación de por lo menos dos registros de representación, y esta coordinación queda de manifiesto por medio del uso rápido y la espontaneidad de la conversión cognitiva" (Duval, 1993, p. 51). Se considera asimismo que la comprensión conceptual surge del desarrollo de la capacidad de coordinación.

\section{Conversión del lenguaje natural al lenguaje algebraico}

Las competencias que intervienen en un proceso de conversión vienen determinadas por los registros fuente e imagen de esa conversión concreta. 
Por ejemplo, en el paso del registro visual al simbólico intervienen procesos de visualización que no se usan necesariamente para la conversión desde lenguaje natural al simbólico. Pero se identifican tres ejes que intervienen con carácter general:

a) La existencia o no existencia de una correspondencia uno a uno entre todas las componentes significativas (símbolos, palabras) de los contenidos de la representación fuente y la representación imagen, es o no posible.

b) El carácter unívoco o no de la elección para cada componente significativa de la representación imagen.

c) Para las componentes significativas que pueden aplicarse, el orden de organización en la representación fuente se mantiene o cambia en la representación imagen. (Duval, 1995, pp. 49-57)

Cuando los tres ejes tienen respuesta afirmativa se dice que la conversión es congruente, denotando esta característica por "C", y no congruente en caso contrario, lo que se denota por "N". En el caso concreto de la conversión del lenguaje natural al algebraico, pensemos en el siguiente ejemplo:

"El conjunto de puntos cuya abscisa y ordenada tienen el mismo signo" que es no congruente con la expresión

$$
\{(x, y): x y>0\} .
$$

Pero esta última sí es congruente con el enunciado siguiente: "El conjunto de puntos tales que el producto de la abscisa por la ordenada es estrictamente mayor que cero".

(Duval, 2006a, pp. 112-113)

Para realizar la conversión en el primer caso es necesario movilizar mentalmente algunos conceptos matemáticos, detectando qué conceptos matemáticos en concreto están involucrados implícitamente en el enunciado verbal e interpretando las propiedades que los relacionan entre sí y con el enunciado. En el ejemplo anterior, esos conceptos son el de número real y producto de números reales, y las relaciones vienen dadas por las propiedades del producto en relación al signo. Vemos también que es insuficiente buscar las llamadas "palabras clave" que remitan a una operación o procedimiento matemático; pueden estar ausentes. 
La parte b) se refiere a la asignación de variables y a la elección de operaciones que reflejen el enunciado verbal. La parte c) se concreta en que la organización sintáctica de las expresiones en el registro fuente e imagen pueden no coincidir y a que las reglas sintácticas del lenguaje natural son diferentes de las del algebraico, donde los símbolos de las operaciones prevalecen sobre los de los números o variables.

\section{Procesamiento de la información en textos matemáticos}

Para la realización de la tarea de conversión propuesta, el primer paso es que el sujeto procese la información presentada en el texto. El modelo cognitivo que seguimos al respecto es el de Kintsch (1998). La comprensión de un texto se concibe como la formación de una imagen mental, siendo esta una red de proposiciones (unidades básicas de significado) relacionadas entre sí y de complejidad variable. A efectos de análisis se distinguen en este proceso dos componentes, el texto-base y la situación-modelo, en función del origen de las proposiciones formadas en la representación mental. Aquellas directamente derivadas del texto, sin añadir nada no explicitado en el mismo, forman el texto-base. Las situaciones-modelo son las proposiciones formadas mediante la integración del texto-base con los conocimientos previos del sujeto para formar una red más coherente. Es en esta componente donde se sitúa la movilización de resultados matemáticos implícitos en el texto para realizar una conversión no congruente que señalábamos en el apartado anterior. El acceso al conocimiento previo se realiza al activarse parte de lo almacenado en la memoria a largo plazo, pasando a formar parte de la "working-memory". Lo anterior nos lleva a considerar también la posible influencia en la tarea de conversión de las variables sintácticas del lenguaje natural, pues por una parte la estructura interna de las proposiciones (de carácter semántico) complejas se relaciona, al menos en parte, con la sintaxis de las oraciones del texto (Kintsch, 1998), y por otra existen estudios sobre la relación entre la complejidad sintáctica y el funcionamiento de la "working memory" (véase Fedorenko, Gibson, \& Rohde, 2007).

\section{Índice de complejidad sintáctica}

Para analizar la complejidad sintáctica de los enunciados en lenguaje natural adaptamos diversos índices en los que intervienen las nociones de 
enunciado y oración, para cuya definición seguimos la gramática de la Real Academia Española de la Lengua (RAE, 2010). Consideramos que un enunciado es la mínima unidad de comunicación que constituye por sí misma un mensaje y es sintácticamente autónoma. Consideramos que una oración es una unidad de predicación que pone en relación un sujeto con un predicado verbal. Diremos que es simple si consta de un solo predicado y compuesta si consta de varios. A su vez, las compuestas se dividen en compuestas por subordinación y por coordinación (se incluyen en este grupo las yuxtapuestas). En el primer caso hay una oración principal y una o varias subordinadas, mientras que en el segundo las oraciones que la componen no presentan dependencia sintáctica entre sí, estando ligadas por un nexo en el caso de las coordinadas y unidas sin partículas intermedias en el caso de las yuxtapuestas. En las oraciones compuestas por subordinación, esta se considera de $1^{\circ}$ grado si todas las oraciones dependen directamente de la principal, de $2^{\circ}$ grado si una oración depende de otra que a su vez depende de la principal, y de $3^{\circ}$ grado si una oración depende de otra subordinada que está subordinada a una subordinada de la principal.

En este estudio adaptamos algunos de los índices introducidos por Salvador (1985). Si bien los ítems del cuestionario de este trabajo constan de un solo enunciado, los índices se definen en general para conjuntos de enunciados. Para adaptarnos a la nomenclatura actual de la RAE, tomamos oraciones donde Salvador (1985) toma proposiciones, y enunciados donde toma oraciones, si bien los valores numéricos que se obtendrían con los índices originales no variarían significativamente.

Índice de complejidad numérica: $\mathrm{CN}:=10 \frac{\mathrm{O}}{\mathrm{E}}$ con $\mathrm{O}$ el número de oraciones y $\mathrm{E}$ el número de enunciados.

Índice de complejidad cualitativa: $\mathrm{CC}:=\frac{\mathrm{S}+2(\mathrm{Y}+\mathrm{C})+3 \mathrm{~S}_{1}+4 \mathrm{~S}_{2}+5 \mathrm{~S}_{3}}{\mathrm{E}}$

con $\mathrm{S}$ el número de oraciones simples, $\mathrm{Y}$ el de yuxtapuestas, $\mathrm{C}$ el de coordinadas, $S_{i}$ el número de subordinadas de grado $i$ y $\mathrm{E}$ el número de enunciados.

Ilustramos con un ejemplo el cálculo de los índices de complejidad sintáctica. 
Enunciado:

Expresa mediante una única condición en lenguaje algebraico la propiedad que cumple un punto del espacio el cual verifica que ninguna de sus coordenadas es nula.

Se cumple que $\mathrm{CC}=5$ pues tenemos una subordinada de grado 3 , siendo las relaciones entre oraciones las siguientes:

"Ninguna de sus coordenadas es nula"

subordinada a

"el cual verifica"

subordinada a

"cumple un punto del espacio".

Diremos que un enunciado es de complejidad sintáctica baja, lo que denotamos por "B", si

$\mathrm{CN} \leq 2, \mathrm{CC} \leq 20$; y alta, denotada por "A", si $C N \geq 40, C \mathrm{C} \geq 5$.

Para establecer estos límites, los inferiores se fijan al constatar los autores la dificultad de redactar enunciados de conversión no congruente para estudiantes universitarios con valores más bajos y tampoco encontrarlos en la literatura; para los superiores se recogen las consideraciones de Salvador (1985) sobre la dificultad práctica de mantener la claridad y coherencia de expresión para valores superiores.

Existen otros índices de complejidad sintáctica, pero escogemos el presente porque tiene en cuenta la organización estructural del enunciado de una forma que se adapta a la estructura de las proposiciones según el esquema predicado-argumentos considerado en Kintsch (1998), identificándose los argumentos con los sintagmas no verbales. Además, en nuestro modelo cognitivo las relaciones entre proposiciones se definen a través de tres niveles de coherencia, siendo similar el tercer nivel a la relación de subordinación oracional (van Dijk \& Kintsch, 1983).

\section{Exposición del problema y objetivos del estudio}

La competencia de manejar estructuras sintácticas complejas, por una parte, y la de movilizar resultados matemáticos implícitos, por otra, parecen a 
priori diferentes, porque un sujeto puede tener un manejo óptimo de estructuras sintácticas complejas pero no ser capaz de movilizar conceptos implícitos por falta de conocimiento matemático. A su vez, amplios conocimientos matemáticos sólo son útiles si el sujeto los sabe relacionar entre sí y con el enunciado espontáneamente. Pero ambas competencias son necesarias en el proceso de conversión, en muchas ocasiones de forma conjunta. Pretendemos entonces responder a las siguientes preguntas.

En la realización de la conversión de enunciados en lenguaje natural a lenguaje algebraico:

- ¿Existen diferencias significativas en función de si la conversión es congruente o no? ¿Y en función de la complejidad sintáctica del enunciado en lenguaje natural?

- ¿Supone una mayor dificultad el carácter no congruente o la complejidad sintáctica alta?

- ¿Existe correlación entre un buen desempeño movilizando conceptos matemáticos implícitos y un buen desempeño manejando estructuras sintácticas complejas?

\section{Metodología del estudio}

\section{Participantes}

En este estudio participaron voluntariamente 46 estudiantes de Grado en Ingeniería de Edificación y 8 del Grado en Ingeniería Informática de una universidad española, todos de primer curso, con edades comprendidas entre los 18 y los 20 años y habiendo seguido la modalidad científico-tecnológica en el bachillerato. Se impuso esta condición para controlar el efecto de la formación inicial. Según los planes de estudio, todos estaban familiarizados con la conversión y conocían los contenidos matemáticos necesarios. En el curso académico en que se llevó a cabo el estudio, la nota de corte para el acceso a esas titulaciones se sitúa entre el 5.23 y el 5.46 .

\section{Variables y instrumento}

Consideramos las variables independientes nominales "tipo conversión" con valores $\mathrm{N}$ y C, y "complejidad sintáctica" con valores A y B; y 
la variable dependiente cuantitativa "puntuación obtenida" con valores en el intervalo [0,4]. Cuando no haya lugar a confusión, llamaremos también $N, C$, A, B, NA, NB, CA y CB, respectivamente, a las variables cuantitativas definidas como la puntuación obtenida por el sujeto en la conversión de un enunciado del tipo correspondiente. A fin de controlar la variabilidad del sujeto, optamos por un diseño intra-sujeto donde cada individuo realiza la conversión de todos los enunciados; estamos, pues, en una situación de medidas repetidas. En las pruebas de contraste de hipótesis, para aceptar o rechazar la hipótesis inicial se toma .05 como valor de referencia para la significatividad asintótica.

\section{Tabla 1 - Ítems tipo NA}

\begin{tabular}{cl}
\hline Ítem & \multicolumn{1}{c}{ Enunciado verbal } \\
\hline 1) & $\begin{array}{l}\text { Expresa mediante una única condición en lenguaje algebraico la propiedad que } \\
\text { cumple un punto del espacio el cual verifica que ninguna de sus coordenadas es } \\
\text { nula. }\end{array}$ \\
\hline 2) & $\begin{array}{l}\text { Expresa mediante una única ecuación la propiedad que cumple una } \\
\text { circunferencia cuyos puntos son tales que equidistan de los puntos de la } \\
\text { circunferencia unidad. }\end{array}$ \\
\hline 3) & $\begin{array}{l}\text { Expresa mediante una única ecuación la propiedad que cumple un número cuyas } \\
\text { tres cifras son tales que si suprimimos la cifra de las centenas entonces el } \\
\text { número que obtenemos es cuatro veces menor que el dado. }\end{array}$ \\
\hline 4$)$ & $\begin{array}{l}\text { Expresa mediante una o varias condiciones en lenguaje algebraico que la relación } \\
\text { entre el ritmo de crecimiento de dos funciones derivables es tal que ambas son } \\
\text { crecientes pero los valores de la primera aumentan a mayor ritmo de lo que lo } \\
\text { hacen los de la segunda. }\end{array}$ \\
\hline 5) & $\begin{array}{l}\text { Expresa con una única ecuación que un punto del espacio verifica que alguna de } \\
\text { sus coordenadas es tal que el siete es un divisor de ella. }\end{array}$ \\
\hline
\end{tabular}

Elaboramos un cuestionario formado por enunciados de los quatro tipos posibles: "NA" (Tabla 1), "NB" (Tabla 2), "CA" (Tabla 3) y "CB" (Tabla 4), que surgen en función de si la conversión es congruente o no y de si la complejidad sintáctica es alta o baja.

Tabla 2 - Ítems tipo NB

\begin{tabular}{cl}
\hline Ítem & \multicolumn{1}{c}{ Enunciado verbal } \\
\hline 1) & $\begin{array}{l}\text { Expresa mediante una única ecuación la siguiente propiedad de una } \\
\text { circunferencia: el centro de la circunferencia equidista de todos los puntos de la } \\
\text { circunferencia unidad. }\end{array}$ \\
\hline 2) & $\begin{array}{l}\text { Expresa con una única ecuación la siguiente propiedad de un punto del espacio: el } \\
\text { siete es un divisor de alguna de sus coordenadas. }\end{array}$ \\
\hline 3) & $\begin{array}{l}\text { Expresa mediante una única condición en lenguaje algebraico la siguiente } \\
\text { propiedad de un punto del espacio: ninguna de sus coordenadas es nula. }\end{array}$ \\
\hline 4) & $\begin{array}{l}\text { Expresa con una única ecuación la siguiente propiedad de un número de tres } \\
\text { cifras: el número sin la cifra de las centenas es otro número cuatro veces menor. }\end{array}$ \\
\hline 5) & $\begin{array}{l}\text { Expresa en lenguaje algebraico la siguiente propiedad para dos funciones } \\
\text { derivables y crecientes: el ritmo de crecimiento de la primera es mayor que el de la } \\
\text { segunda. }\end{array}$ \\
\hline
\end{tabular}


Para limitar la influencia de la mayor o menor familiarización de cada sujeto con un contenido matemático concreto, en el cuestionario cada concepto y propiedad aludida en un tipo de ítem también aparece en los otros, si bien de forma desordenada y siempre primero en el NA. Además, los ítems en conjunto incluyen:

- Varios campos de referencia: números naturales [en 3), 9), 15) y 20)], números enteros [en 5), 7), 12) y 17)], números reales [en 1), 8), 14), 19) y 18)], teoría de funciones [en 4), 10), 13) y 16)], geometría analítica [en 2), 6) y 11)].

- Casos donde alguna variable se usa como parámetro [en 2), 5), 6), 7), 11), 12) y 17)] y otros donde no se usa.

- Casos donde el contenido geométrico es relevante en la conversión para determinar el número de variables (en 1), 2), 5), 6), 7), 8), 11) y 12)], otros donde es necesario movilizar resultados geométricos [en 2) y 6)], y otros donde no influye o no está presente.

\section{Tabla 3 - Ítems tipo CA}

\begin{tabular}{cl}
\hline Ítem & \multicolumn{1}{c}{ Enunciado verbal } \\
\hline 1) & $\begin{array}{l}\text { Escribe una ecuación que caracterice los puntos del plano para los cuales existe } \\
\text { un número cuyo cuadrado es igual a la suma del cuadrado de la primera } \\
\text { coordenada del punto más el cuadrado de la segunda. }\end{array}$ \\
\hline 2) & $\begin{array}{l}\text { Expresa mediante una ecuación que un punto del espacio tiene la propiedad de } \\
\text { que si realizamos el producto de sus coordenadas entonces el resultado es igual } \\
\text { a tres multiplicado por un número. }\end{array}$ \\
\hline 3) & $\begin{array}{l}\text { Expresa mediante una o varias condiciones en lenguaje algebraico que la relación } \\
\text { entre el ritmo de crecimiento de dos funciones derivables es tal que las derivadas } \\
\text { de ambas son positivas pero la derivada de la primera es mayor que la derivada } \\
\text { de la segunda. }\end{array}$ \\
\hline 4) & $\begin{array}{l}\text { Expresa mediante una condición en lenguaje algebraico que un punto del espacio } \\
\text { verifica que el resultado del producto de sus tres coordenadas es un número que } \\
\text { es distinto de cero. }\end{array}$ \\
\hline 5) & $\begin{array}{l}\text { Expresa mediante una ecuación la propiedad que cumple un número cuyas tres } \\
\text { cifras son tales que la cifra de las unidades más la de las decenas por diez más la } \\
\text { de las centenas por cien es igual a cuatro veces la suma de la cifra de las } \\
\text { unidades más diez por la de las decenas. }\end{array}$ \\
\hline
\end{tabular}

Un cuestionario inicial con 64 enunciados fue sometido a un panel de expertos formado por licenciados en matemáticas, docentes en educación secundaria y universitaria. Se preguntó sobre la idoneidad, claridad y dificultad de cada ítem, la cantidad, y el tiempo necesario para que un alumno medio realizara cada conversión. Teniendo en cuenta su opinión se seleccionaron 24 enunciados y se modificó la redacción. A continuación, se 
realizó un estudio piloto con ocho estudiantes para calibrar la dificultad y la aparición del cansancio. A partir de los resultados se redujo el número de ítems a 20, cinco de cada tipo (Tablas 1, 2, 3 y 4), desechando los estadísticamente no significativos. El orden de presentación de los enunciados en la prueba definitiva sigue la numeración indicada en las Tablas $1,2,3$ y 4 .

\section{Tabla 4 - Ítems tipo CB}

\begin{tabular}{cl}
\hline Ítem & \multicolumn{1}{c}{ Enunciado verbal } \\
\hline 1) & $\begin{array}{l}\text { Expresa la siguiente relación entre dos funciones derivables: la derivada de } \\
\text { la primera función es mayor que la derivada de la segunda. }\end{array}$ \\
\hline 2) & $\begin{array}{l}\text { Expresa mediante una ecuación la siguiente propiedad de un punto del } \\
\text { espacio: el producto de sus tres coordenadas es igual a cuatro multiplicado } \\
\text { por otro número. }\end{array}$ \\
\hline 3) & $\begin{array}{l}\text { Expresa mediante una condición en lenguaje algebraico la siguiente } \\
\text { propiedad de un punto del espacio: el producto de sus tres coordenadas es } \\
\text { distinto de cero. }\end{array}$ \\
\hline 4$)$ & $\begin{array}{l}\text { Expresa mediante una ecuación la siguiente propiedad de tres variables: el } \\
\text { cuadrado de la primera más el cuadrado de la segunda es igual al cuadrado } \\
\text { de la tercera. }\end{array}$ \\
\hline 5) & $\begin{array}{l}\text { Expresa mediante una ecuación la siguiente propiedad de un número de } \\
\text { tres cifras: la suma de la cifra de las decenas multiplicada por diez más la } \\
\text { cifra de las unidades, todo esto multiplicado por 4, es igual a la cifra de las } \\
\text { centenas multiplicada por cien, más la cifra de las decenas multiplicada por } \\
\text { diez, más la cifra de las unidades. }\end{array}$ \\
&
\end{tabular}

Para evitar equívocos se impuso la condición de no usar cuantificadores en las expresiones algebraicas; de lo contrario, algunos enunciados $\mathrm{N}$ pasarían a ser $\mathrm{C}$. Por ejemplo, el ítem 1) podría considerarse de conversión directa si escribimos

$$
\mathrm{x}_{i} \neq 0 \forall \mathrm{i} \in\{1,2,3\} .
$$

Para categorizar las respuestas, primero se analizó cada ítem para comprobar que se correspondía con el tipo asignado y se contrastó la clasificación con el criterio del panel de expertos. Incluimos en la Tabla 5 los conceptos y propiedades matemáticos implícitos en los de tipo $\mathrm{N}$. 
Tabla 5 - Propiedades, conceptos y expresión correcta por ítem

\begin{tabular}{|c|c|c|}
\hline Ítem & & \\
\hline \multirow[t]{2}{*}{ 1), 8) } & $\begin{array}{l}\text { Conceptos, } \\
\text { propiedades }\end{array}$ & $\begin{array}{l}\text { El producto de dos números reales es distinto de } \\
\text { cero si, y solo si, ambos lo son }\end{array}$ \\
\hline & Expresión & $x y z \neq 0$ \\
\hline \multirow[t]{2}{*}{ 2), 6) } & $\begin{array}{l}\text { Conceptos, } \\
\text { propiedades }\end{array}$ & $\begin{array}{l}\text { Circunferencia; el centro de la circunferencia es el } \\
\text { único punto del plano que equidista de todos los } \\
\text { puntos de dicha circunferencia; los puntos de una } \\
\text { circunferencia equidistan de los de otra si, y solo } \\
\text { si, el centro de ambas es el mismo; los puntos de } \\
\text { cualquier circunferencia con centro en el origen } \\
\text { equidistan de la circunferencia unidad }\end{array}$ \\
\hline & Expresión & $x^{2}+y^{2}=p^{2}$ \\
\hline \multirow[t]{2}{*}{ 3), 9) } & $\begin{array}{l}\text { Conceptos, } \\
\text { propiedades }\end{array}$ & Sistema posicional decimal de numeración \\
\hline & Expresión & $(10 b+a) 4=100 c+10 b+a$ \\
\hline \multirow[t]{2}{*}{ 4), 10) } & $\begin{array}{l}\text { Conceptos, } \\
\text { propiedades }\end{array}$ & $\begin{array}{l}\text { Derivada de una función; interpretación de la } \\
\text { derivada de una función en un punto como tasa } \\
\text { de crecimiento instantáneo; estudio del } \\
\text { crecimiento de una función derivable a través del } \\
\text { signo de su derivada }\end{array}$ \\
\hline & Expresión & $f^{\prime}>g^{\prime}>0$ \\
\hline \multirow[t]{2}{*}{ 5), 7) } & $\begin{array}{l}\text { Conceptos, } \\
\text { propiedades }\end{array}$ & $\begin{array}{l}\text { Divisibilidad; el número } 5 \text { es primo; un número } \\
\text { primo divide a un producto si, y solo si, divide a } \\
\text { alguno de los factores }\end{array}$ \\
\hline & Expresión & $x y z=7 d$ \\
\hline
\end{tabular}

Antes de la prueba piloto se estableció una primera categorización de las posibles respuestas, pero esta fue modificada después de analizar las respuestas reales, adjudicándose un valor en $[0,1]$ según los siguientes criterios:

1 Asignación de variables y/o símbolos.

2 Identificación de conceptos matemáticos y sus propiedades (implícitas o explícitas) presentes en el enunciado.

3 Identificación y uso correcto de operaciones y relaciones algebraicas.

4 Expresión formal correcta.

La puntuación asignada a cada sujeto para un tipo de ítem es la media aritmética de sus puntuaciones correspondientes a los cinco ítems de ese tipo.

Ilustramos los criterios de corrección con algunos ejemplos (puntuación incluida en la figura). 
1)

$x y z>0$

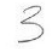

Figura 1 - Respuesta del estudiante 20 al ítem 1)

En la Figura 1 el sujeto asigna correctamente variables, una para cada coordenada, moviliza las propiedades adecuadas (Tabla 5) no explicitadas en el enunciado y usa correctamente las operaciones algebraicas, pero la expresión final es incorrecta pues no considera los casos donde el producto es estrictamente menor que cero.

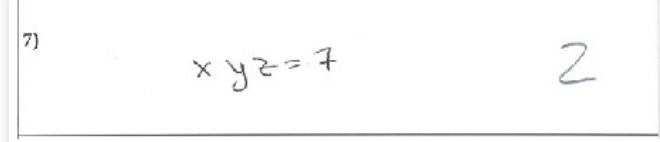

Figura 2 - Respuesta del estudiante 32 al ítem 7)

En la Figura 2 el sujeto identifica tres de las cuatro variables y sólo una de las dos operaciones implicadas. Si bien su respuesta parece no tener sentido, interpretamos que el sujeto moviliza, al menos hasta cierto punto, la propiedad consistente en que un número primo divide a un producto si, y solo si, divide a alguno los factores, así como que el 7 es primo. Al mencionarse la divisibilidad en el enunciado interpretamos que el sujeto la tiene en cuenta y que el error proviene de falta de dominio del lenguaje algebraico.

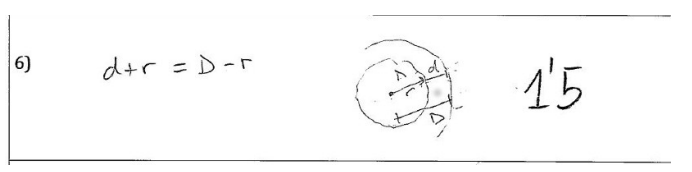

Figura 3 - Respuesta del estudiante 29 al ítem 6) 
En la Figura 3, en un ítem que alude a conceptos geométricos, el sujeto usa un tercer registro semiótico como puente entre lenguaje natural y algebraico. Esto parece ayudarle a movilizar mentalmente conceptos, pues la figura plasma la propiedad de que la circunferencia pedida tiene el mismo centro que la circunferencia unidad. Por ello, interpretamos que la expresión algebraica es incorrecta debido a un dominio insuficiente del lenguaje algebraico o a la falta de coordinación entre los registros gráfico y algebraico.

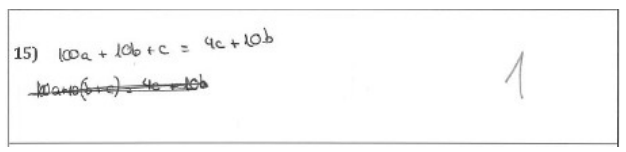

Figura 4 - Respuesta del estudiante 33 al ítem 15)

En la Figura 4 se asignan correctamente variables pero no se identifican bien todas las operaciones ni se capta la propiedad distributiva.

\section{Procedimiento}

La prueba definitiva fue administrada por los autores en el aula habitual de los grupos. La semana previa, los autores comunicaron a los estudiantes su intención de aplicar el cuestionario, indicando que la participación sería voluntaria y anónima. Antes de pasar el cuestionario se explicó con detalle a los participantes en qué consistía la tarea.

Cada enunciado se mostró de 2 a 3 minutos en una pantalla situada en la cabecera del aula; al cabo de este tiempo se sustituía por el siguiente para evitar el empleo de demasiado tiempo en un ítem y que eso interfiriese en ítems posteriores. La duración total fue de 55 minutos.

Una vez obtenidas las puntuaciones definitivas, la prueba de bondad de ajuste de Kolmorov-Smirnov indica ausencia de normalidad, por lo que no realizamos un ANOVA con medidas repetidas en ambos factores. Esto nos restringe a estudios con medidas repetidas y un factor.

En función de la verificación o no de los supuestos de normalidad y simetría, usamos la prueba t-Student para muestras relacionadas, la de Wilcoxon o la de los signos para comparar promedios, y el coeficiente de correlación de Pearson o el de Spearman para relacionar variables. 
Para testar la simetría mediante un índice tipificado, dividimos el índice de asimetría entre su error típico y consideramos la distribución simétrica si su valor absoluto es estrictamente menor que dos. Para medir el tamaño del efecto en el caso no paramétrico usamos el índice

$$
\delta=\frac{\left|\mu_{1}-\mu_{2}\right|}{\sigma_{D}}
$$

donde $\mu_{i}$ es la media muestral de la muestra i, y $\sigma_{\mathrm{D}}$ es la desviación típica de la variable diferencia.

\section{Resultados}

Como primera aproximación mostramos los descriptivos en la Tabla 6.

Tabla 6 - Estadísticos descriptivos

\begin{tabular}{lllll}
\hline & Mínimo & Máximo & Media & Desv. típica \\
\hline NA & 0.00 & 2.20 & 0.8435 & 0.53982 \\
\hline NB & 0.00 & 2.40 & 0.9174 & 0.56502 \\
\hline CA & 0.20 & 4.00 & 2.4435 & 1.01448 \\
\hline CB & 2.40 & 4.00 & 3.5043 & 0.54933 \\
\hline N & 0.00 & 2.20 & .8804 & 0.49693 \\
\hline C & 1.40 & 4.00 & 2.9739 & 0.66714 \\
\hline A & 0.20 & 2.50 & 1.6435 & 0.59987 \\
\hline B & 1.30 & 3.20 & 2.2109 & .44832 \\
\hline
\end{tabular}

En cuanto a los contrastes de hipótesis sobre simetría de las distribuciones, los resultados indican que el valor absoluto del índice tipificado es menor que dos en todos los pares excepto en $(C A, C B)$, donde para comparar los promedios usamos la prueba de los signos, que con una significatividad .001 nos lleva a rechazar la hipótesis nula de igualdad de medianas, con tamaño del efecto $\delta=1.13$. Por la prueba de KolmogorovSmirnov, usamos la prueba de Wilcoxon para los pares (NA,CA), (NA,NB) y $(\mathrm{NB}, \mathrm{CB})$. Empleamos la t-Student para $(\mathrm{N}, \mathrm{C})$ y $(\mathrm{A}, \mathrm{B})$. Por la prueba de Wilcoxon (Tabla 7 ) rechazamos la igualdad de medianas para (NA,CA), (NB,CB). 
Tabla 7 - Prueba de Wilcoxon

\begin{tabular}{|c|c|c|c|}
\hline & NB - NA & $\mathrm{CA}-\mathrm{NA}$ & $\mathrm{CB}-\mathrm{NB}$ \\
\hline Z & -0.898 & -5.435 & -5.913 \\
\hline$p^{a}$ & .369 & .000 & .000 \\
\hline$\delta$ & & 1.45 & 3.91 \\
\hline
\end{tabular}

Los resultados de la prueba t-Student indican el rechazo de la igualdad de medias en ambos pares. En concreto, para $(\mathrm{N}, \mathrm{C})$ obtenemos $\mathrm{t}(45)=19.87$, $\mathrm{p}<.00,95 \%$ IC [1.88, 2.31], con media de la diferencia 2.09 y desviación típica 0.72 . Para el par $(A, B)$, con una media de la diferencia de 0.57 y desviación típica 0.45 , resulta $\mathrm{t}(45)=7.72, \mathrm{p}<.00,95 \% \mathrm{IC}[0.42,0.72]$.

Sobre la correlación, al tener una expectativa a priori de relación creciente, aplicamos contrastes unilaterales. En el caso paramétrico, obtenemos en todos los pares una relación lineal significativa (Tabla 8). En el caso no paramétrico, obtenemos una relación monótona creciente en los pares incluidos en las tres últimas filas de la Tabla 8, mientras que aceptamos la hipótesis nula en los casos (NA,CA), (NA,CB) y (NB,CA).

Tabla 8 - Coeficientes de correlación

\begin{tabular}{lccccccccc}
\hline Par & $(\mathrm{N}, \mathrm{C})$ & $(\mathrm{N}, \mathrm{A})$ & $(\mathrm{N}, \mathrm{B})$ & $(\mathrm{C}, \mathrm{A})$ & $(\mathrm{C}, \mathrm{B})$ & $(\mathrm{A}, \mathrm{B})$ & $(\mathrm{NA}, \mathrm{NB})$ & $(\mathrm{NB}, \mathrm{CB})$ & $(\mathrm{CA}, \mathrm{CB})$ \\
\hline $\mathrm{r}$ & 0.27 & 0.58 & 0.73 & 0.86 & 0.64 & 0.58 & & & \\
\hline $\mathrm{r}$ & & & & & & & 0.56 & 0.28 & 0.47 \\
\hline $\mathrm{p}$ & 0.033 & .00 & .00 & .00 & .00 & .00 & .000 & .032 & .000 \\
\hline $\mathrm{r}=$ coeficiente de correlación de Pearson; $\mathrm{r}_{\mathrm{S}}=$ coeficiente de Spearman; $\mathrm{p}=$ significatividad unilateral \\
\hline
\end{tabular}

\section{Conclusiones y discusión}

Los resultados indican que al variar el carácter congruente o no del enunciado las diferencias en las medias de las puntuaciones son estadísticamente significativas en todos los casos; mientras que al variar la complejidad sintáctica lo son siempre excepto al restringirnos a enunciados $\mathrm{N}$. Entonces contestamos afirmativamente a la primera pregunta propuesta en los objetivos, si bien el hecho de que varíen siempre excepto en (NA,NB) sugiere que la complejidad sintáctica es una dificultad pero de distinto orden que el carácter no congruente, siendo este último lo determinante, en concordancia con lo observado en Duval (1995, 2006a, 2006b). Relacionado 
con esto, el tamaño del efecto y los intervalos de estimación de las diferencias entre medias también corroboran que el tamaño del efecto es mayor para el cambio en la congruencia que para la complejidad sintáctica, lo que responde a la segunda pregunta de los objetivos. Otro aspecto importante es que el tamaño del efecto pasa de 1.45 para $(\mathrm{NA}, \mathrm{CA})$ a 3.91 para $(\mathrm{NB}, \mathrm{CB})$. Entonces, los enunciados de complejidad sintáctica baja hacen más visibles las dificultades para realizar conversiones no congruentes. Esto tiene implicaciones didácticas, pues la dificultad en el procesamiento de la información que supone una complejidad sintáctica alta impide al docente distinguir si el estudiante no tiene la competencia de movilizar espontáneamente determinado contenido o bien el problema está solo en el procesamiento del lenguaje natural. Estas consideraciones sugieren la importancia de tener en cuenta la complejidad sintáctica del lenguaje en las actividades de aula, pues es verosímil que dificultades del tipo de las observadas en tareas aisladas de conversión se manifiesten también en cualquier otra actividad de construcción de conocimiento y evaluación. Otro motivo para no minusvalorar la complejidad sintáctica es que el tamaño del efecto en $(C A, C B)$ es próximo al de (NA,CA).

En cuanto a la tercera pregunta de los objetivos, si bien los coeficientes de Pearson (Tabla 8) muestran relación significativa en todos los casos, el coeficiente para $(\mathrm{N}, \mathrm{C})$ es mucho menor que en los otros pares. Además, en el caso no paramétrico, si las componentes del par comparado varían en cuanto a congruencia, o bien no existe relación o esta es mucho menor que cuando la congruencia no varía. Dada la variedad de contenidos impuesta en el diseño del cuestionario y a la vista de los resultados empíricos, las diferencias parecen deberse a la no congruencia en sí misma, y no a un contenido matemático o a un campo de referencia concretos. Estas observaciones sugieren una gran diferencia a nivel cognitivo entre la realización de conversiones congruentes y no congruentes.

Si concebimos, en la línea de Duval (1995, 2006a, 2006b) o Dreyfus (1991), la capacidad para cambiar de registro de representación como un indicador de la comprensión, los resultados (Tabla 6) sugieren un déficit importante al respecto para los contenidos del cuestionario, pues estos se suponen bien conocidos para estudiantes de primer curso de Ingeniería, como los participantes en la prueba. 


\section{Referências}

Dreyfus, T. (1991). Advanced mathematical thinking processes. In D. Tall (Ed.), Advanced mathematical thinking (pp. 25-41). Netherlands: Kluvert Academic Publishers.

Duval, R. (1993). Registres de représentations sémiotiques et fonctionnement cognitif de la pensée. Annales de Didactique et de Sciences Cognitives, 5, 37-65.

Duval, R. (1995). Sémiosis et pensée humaine. Registres sémiotiques et apprentissages intellectuels. Berna: Peter Lang.

Duval, R. (2004). Los problemas fundamentales en el aprendizaje de las matemáticas y las formas superiores del desarrollo cognitivo. Cali: Universidad del Valle.

Duval, R. (2006a). A cognitive analysis of problems of comprehension in a learning of mathematics. Educational Studies in Mathematics, 61(1-2), 103-131.

Duval, R. (2006b). Un tema crucial en la educación matemática: La habilidad para cambiar de registro de representación. La Gaceta de la Real Sociedad Matemática Española, 9(1), 143-168.

Fedorenko, E., Gibson, E., \& Rohde, D. (2007). The nature of working memory in linguistic, arithmetic and spatial integration processes. Journal of Memory and Language, 56, 246-269.

Golding, G. (1998a). Representational systems, learning, and problem solving in mathematics. Journal of Mathematical Behaviour, 17(2), 137-165.

Golding, G. (1998b). The PME Working Group on representations. Journal of Mathematical Behaviour, 17(2), 283-301.

Kaput, J. (1987). Towards a theory of symbol use in mathematics. In C. Janvier (Ed.), Problems of representation in mathematics learning and problem solving (pp. 159-196). Hillsdale, USA: Lawrence Erlbaum Associates.

Kintsch, W. (1998). Comprehension. A paradigm for cognition. Cambridge: Cambridge University Press.

Pimm, D. (1987). Speaking mathematically: Communication in mathematics classrooms. London: Routledge \& Kegan Paul.

Puig, L., \& Cerdán, F. (1988). Problemas aritméticos escolares. Madrid: Síntesis.

Real Academia Española de la Lengua (2010). Nueva gramática de la lengua española. Manual. Madrid: Espasa.

Salvador F. (1985). Los índices de complejidad sintáctica, instrumentos de evaluación de la expresión escrita: Estudio experimental en el ciclo medio de EGB. Enseñanza, 3, 59-81.

Shapiro, S. (1997). Philosophy of mathematics. Structure and ontology. Oxford: Oxford University Press.

Valentin, J. D., \& Chap-Sam, L. (2005). Roles of semantic structure of arithmetic word problems on pupils' ability to identify the correct operation. International Journal for Mathematics Teaching and Learning, May, 1-14. Disponible en: http://www.cimt.org.uk/journal/valentin.pdf 
Van Dijk, T. A., \& Kintsch, W. (1983). Strategies of discourse comprehension. New York: Academic Press.

Villegas, J. L., Castro, E., \& Gutiérrez, J. (2009). Representaciones en resolución de problemas: Un estudio de caso con problemas de optimización. Electronic Journal of Research in Educational Psychology, 7(17), 279-308. Disponible en: http://www.redalyc.org/articulo.oa?id=293121936015 


\section{COMPETÊNCIAS DIFERENCIADAS NO PROCESSO DE CONVERSÃO DA LINGUAGEM NATURAL PARA A ALGÉBRICA}

\section{Resumo}

O uso de diferentes registos semióticos e a mudança de um para outro são características da atividade cognitiva na matemática, e as dificuldades associadas a estas mudanças são uma das fontes de mal entendidos e dificuldades na aprendizagem. No caso da mudança de linguagem natural para algébrica intervêm, no mínimo, dois tipos de competências: gerir estruturas sintáticas na linguagem verbal e movimentar mentalmente conceitos matemáticos implícitos nos enunciados. A fim de estudar a incidência de cada uma delas, as dificuldades que apresentam e as interrelações entre si, elaborámos um questionário, que aplicámos a um grupo de caloiros de Engenharia, e analisamos os resultados por meio de testes de hipóteses, apurando conclusões aplicáveis ao padrão de instrução. Os resultados mostram diferenças estatisticamente significativas em função do chamado carácter congruente ou não da mudança de registo e também da complexidade sintática do enunciado.

\section{Palavras-chave}

Linguagem Verbal; Linguagem Algébrica; Conversão; Complexidade Sintática

DIFFERENT COMPETENCIES IN THE CONVERSION PROCESS FROM NATURAL TO ALGEBRAIC LANGUAGE

\section{Abstract}

The use of different semiotic registers and the change of register are characteristics of the cognitive activity in mathematics, and the difficulties associated to these changes a source of incomprehension and learning difficulties. In the conversion process from the natural to the algebraic language, at least two types of cognitive skills are involved: to handle syntactic 
structures in natural language, and to mentally mobilize the mathematical concepts expressed in the text. In order to study the relevance of each skill, the associated difficulties and interrelationship, we designed a questionnaire, handed it out to a group of Engineering students, and analyzed the results by means of statistical hypothesis testing, from which we obtained conclusions applicable to teaching design. The results show differences depending on the so-called congruence of the semiotic register change, as well as on the verbal statement of syntactic complexity.

Keywords

Verbal Language; Algebraic Language; Conversion; Syntactic Complexity

Recebido em outubro 2016

Aceite para publicação em outubro 2017

i Depto. de Pedagoxía e Didáctica, Facultade de Ciencias da Educación, Universidade da Corunha, España.

ii Depto. de Computación, Facultade de Informática, Universidade da Coruña, España.

iii Depto. de Matemáticas, Escola Universitaria de Arquitectura Técnica, Universidade da Coruña, España.

Toda a correspondência relativa a este artigo deve ser enviada para: Carlos Soneira Calvo, Depto. de Pedagoxía e Didáctica, Universidade da Corunha, Facultade de Ciencias da Educación, Campus de Elviña, 15071 A Corunha, España. E-mail: carlos.soneira@udc.gal 